\title{
Nivel de conocimiento sobre técnica del electrocardiograma relacionado con interpretación básica por estudiantes de enfermería
}

\author{
Hurtado-de la Cruz Diana Cecilia*, Moreno-Ortega Diana Janinne*, Padilla- Briones Zandra
}

Giselle*, Reyes-Leos Juan Carlos*, Rojas-González Carolina Elizabeth*, Guerrero-Mojica Nery**

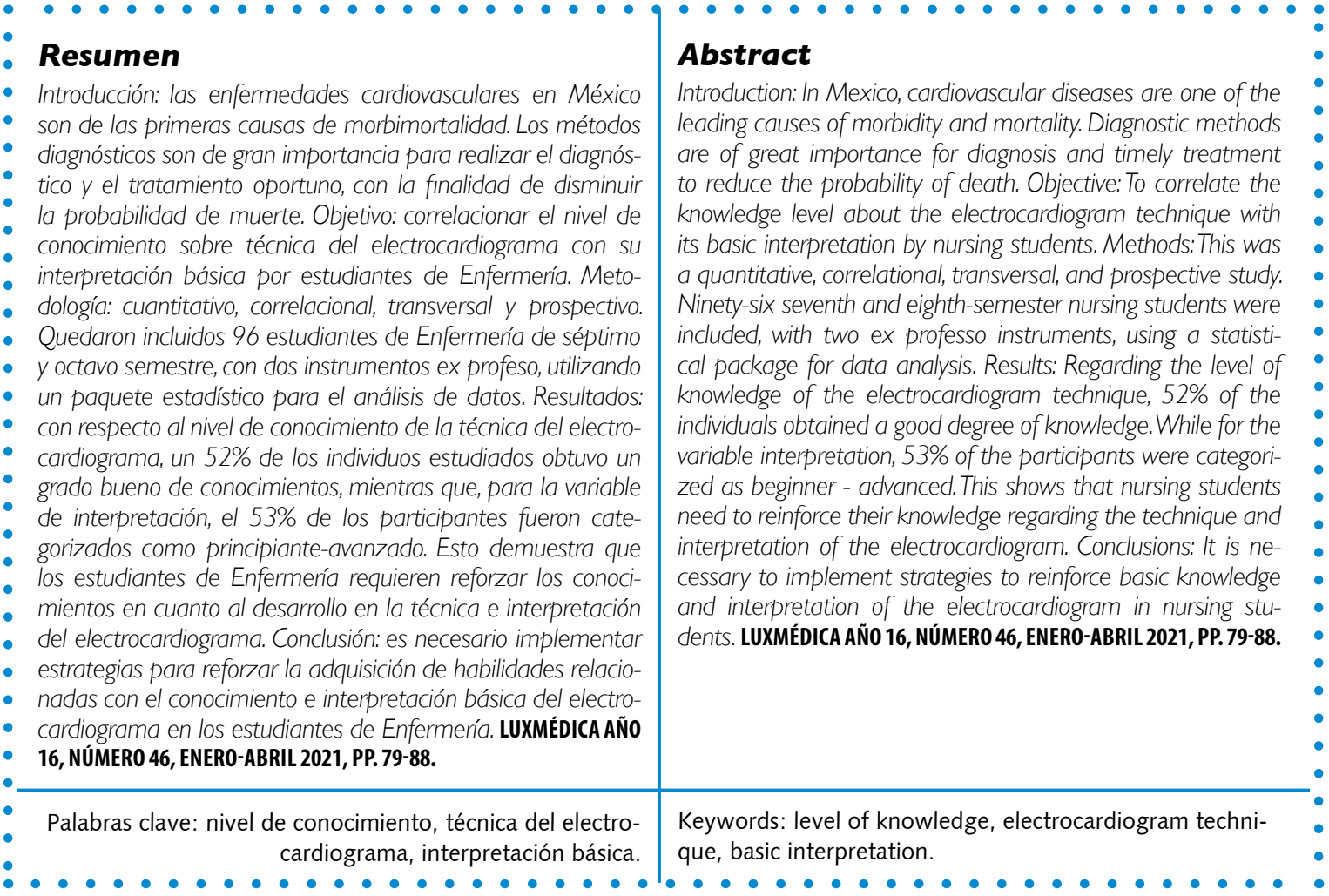

Hurtado-de la Cruz Diana Cecilia. Estudiante de la Licenciatura de Enfermería del Centro de Ciencias de la Salud de la Universidad Autónoma de Aguascalientes. Correo electrónico ceciihurtado59@gmail.com https://orcid.org/0000-0001-6047-6156

Moreno-Ortega Diana Janinne. Estudiante de la Licenciatura de Enfermería del Centro de Ciencias de la Salud de la Universidad Autónoma de Aguascalientes. Correo Moreno-Ortega D

electrónico djaninne_moor3@hotmail.com https://orcid.org/0000-0002-0501-3260

Padilla-Briones Zandra Giselle. Estudiante de la Licenciatura de Enfermería del Centro de Ciencias de la Salud de la Universidad Autónoma de Aguascalientes. Correo electrónico zandrapadillabriones@gmail.com https://orcid.org/0000-0002-6042-7773

Reyes-Leos Juan Carlos. Estudiante de la Licenciatura de Enfermería del Centro de Ciencias de la Salud de la Universidad Autónoma de Aguascalientes. Correo electrónico amigo1386@hotmail.com https://orcid.org/0000-0002-3797-8040

Rojas-González Carolina Elizabeth. Estudiante de la Licenciatura de Enfermería del Centro de Ciencias de la Salud de la Universidad Autónoma de Aguascalientes. Correo electrónico carolina.rojascerg@gmail.com https://orcid.org/0000-0003-0308-5911

Guerrero-Mojica Nery. Profesora Investigadora del Departamento de Enfermería del Centro de Ciencias de la Salud de la Universidad Autónoma de Aquascalientes. Correo electrónico g.n.ery@hotmail.com https://orcid.org/0000-0003-0580-4086

Fecha de recibido: 30 de enero 2020

Fecha de aceptación: 2 de septiembre 2020

Correspondencia: MCE Nery Guerrero Mojica. Departamento de Enfermería. Edificio 105 Ciudad Universitaria, Avenida Universidad \# 940. Código postal 20131. Aguascalientes, Aguascalientes, México. Teléfono 449-910-84-37 Correo electrónico g.n.ery@hotmail.com 


\section{Introducción}

Las enfermedades cardiovasculares representan una de las principales causas de morbimortalidad en México, de tal manera que en la actualidad los métodos diagnósticos son de gran importancia para detectar alguna patología cardiovascular y brindar cuidados oportunos. A nivel mundial según la Organización Mundial de la Salud (OMS), las enfermedades cardiovasculares son la principal causa de muerte, se calcula que en 2015 murieron por esta razón 17.7 millones de personas.

De estas muertes, 7.4 millones se debieron a la cardiopatía coronaria, y 6.7 millones, a los accidentes cerebrovasculares (ACV), al menos tres cuartas partes de las defunciones causadas por ACV en el mundo se producen en los países de ingresos bajos y medios donde han superado a las enfermedades infecciosas como primera causa de muerte y su impacto amenaza al desarrollo social y económico de estas regiones. ${ }^{1}$

Aunque las enfermedades crónicas afectan principalmente a las personas de edad avanzada casi la mitad de las muertes por enfermedades crónicas se producen prematuramente, en personas menores de 70 años y una cuarta parte de esas defunciones se dan en personas menores de 60 años. ${ }^{2}$ Estas enfermedades causaron en el 2013 más de 17.3 millones de muertes, una cifra que se proyecta aumentará a más de 23.6 millones para el año 2030. ${ }^{3}$

La cardiopatía afecta sobre todo a los hombres de mediana y avanzada edad y su mortalidad es $20 \%$ más alta en los hombres que en las mujeres. ${ }^{4}$ Representa un factor importante de muerte en todos los adultos, el mayor número de defunciones ocurre en personas de ambos géneros de 65 años o más, lo que subraya que la edad avanzada es un poderoso inductor de esta enfermedad. ${ }^{5}$

El electrocardiograma es un método de utilidad diagnóstica basado en el registro de la actividad eléctrica cardiaca. El corazón, para contraerse y ejercer su función, necesita estimularse eléctricamente; estos estímulos eléctricos producen diferencias de potencial, que pueden registrarse en un papel milimétrico para su posterior interpretación. ${ }^{6}$

Como referente a los conocimientos sobre electrocardiograma del personal de salud, se registra un estudio realizado en Lima, Perú, a las enfermeras del Hospital Arzobispo Loayza, que midió el nivel de conocimiento sobre la valoración del electrocardiograma y su registro en las notas de enfermería del personal de enfermería de la Unidad Coronaria comparado con el personal de Cuidados Intensivos. Se demostró que el personal de enfermería de la unidad coronaria tiene un nivel de conocimiento bueno en el $76.46 \%$, pero no lleva un buen registro en las notas de enfermería, mientras que las enfermeras de cuidados intensivos tienen un nivel de conocimiento bueno y realizan un buen registro en las notas de enfermería (eficiente 100\%).? 
Estadísticas nacionales refieren que el 19\% de mujeres y hombres de 30 a 69 años muere por enfermedades cardiovasculares. El Sistema Nacional de Salud afirma que la cardiopatía isquémica y la diabetes mellitus son dos de los más grandes problemas de la salud pública. ${ }^{8,9}$ Por lo que un estudio realizado a estudiantes becarios de Ciencias de la Salud, implementado en distintos grados en la Universidad de Monterrey, demuestra la aptitud clínica en la interpretación de electrocardiograma. Se encontró que las calificaciones más altas fueron de 44 aciertos, siendo el esperado máximo de 77 aciertos, teniendo como conclusión que los participantes tienen un pobre conocimiento en la interpretación del ECG. ${ }^{10}$ En Aguascalientes, según estadísticas del Instituto Nacional de Estadística y Geografía (INEGI) 2015, las principales causas de muerte son las enfermedades del corazón. ${ }^{11,12} \mathrm{Al}$ menos el $60 \%$ de los habitantes de Aguascalientes que han sido atendidos en las jornadas de salud del Sistema Nacional para el Desarrollo Integral de la Familia (DIF) presentan indicios de enfermedades cardiovasculares o renales, lo que indica una problemática de salud importante que debe ser atendida por las instancias de salud..$^{13}$

Durante el año 2015 se realizó un estudio a las Enfermeras de un reconocido hospital del estado, sobre el nivel de conocimiento en el monitoreo del electrocardiograma, obteniendo como resultado que tienen mayor conocimiento en la anatomía del corazón, pero un bajo nivel en la interpretación de los trazos en el electrocardiograma. ${ }^{14}$

El Instituto Mexicano del Seguro Social (IMSS) en Aguascalientes implementará un nuevo Modelo Preventivo, cuyo objetivo es evitar que los trabajadores sanos desarrollen enfermedades cardiovasculares, diabetes, cáncer de mama y de próstata, por ser consideradas las principales causas de muerte en el IMSS. ${ }^{15}$

La prevención de enfermedades cardiovasculares es de suma importancia debido a su índice de mortalidad en el estado de Aguascalientes, abordando los factores de riesgo modificables en la población. ${ }^{16}$

En cuanto a conceptos disciplinares relacionados con la teoría del nivel de conocimiento basado en Patricia Benner, describe las características de la actuación enfermera en las diferentes etapas de adquisición de habilidades y sustenta que la práctica puede ampliar los conocimientos y habilidades del profesional a través del tiempo. Según la teoría de Patricia Benner, la adquisición de habilidades por el personal de Enfermería comprende de cinco etapas las cuales se describirán a continuación: ${ }^{17}$

a. Principiante: es la persona que no tiene ninguna experiencia previa de la situación a la que debe enfrentarse, en este estadio se encuentran los estudiantes de Enfermería, aunque también podrán encontrarse las enfermeras expertas en un área determi- 
nada cuando tienen que enfrentarse a una situación que les es desconocida. ${ }^{17}$

b. Principiante - avanzada: es la persona que puede demostrar una actuación aceptable por lo menos parcialmente después de haberse enfrentado a un número suficiente de situaciones reales o después de que un tutor le haya indicado los elementos importantes recurrentes de la situación. En este estadio, la persona posee la experiencia necesaria para dominar algunos aspectos de la situación, aunque tienen problemas para dominar la situación actual del paciente desde una perspectiva más amplia. Aquí se incluye la enfermera egresada. ${ }^{17}$

c. Competente: este nivel se caracteriza por una considerable planificación consistente y deliberada que determina los aspectos de las situaciones actuales y futuras que son importantes, teniendo la capacidad de limitar las acciones que hacen sus colegas de las situaciones reales, y empieza a reconocer los patrones. A esta etapa pertenecen las enfermeras que tienen 2 o 3 años de experiencia laboral. ${ }^{17}$

d. Eficiente: la enfermera tiene una experiencia de 3 a 5 años; es capaz de reconocer los aspectos más importantes del paciente y posee un dominio intuitivo de la situación a partir de la información previa que conoce sin perder el tiempo en soluciones alternativas. ${ }^{17}$

e. Experta: posee un dominio intuitivo de la situación y es capaz de identificar el origen del problema. La enfermera experta posee habilidad de reconocer patrones gracias a su amplia experiencia laboral con más de 5 años. La enfermera desarrolla su ejercicio profesional de manera flexible y muy eficiente; ya no necesita reglas, directrices o máximas para conectar su conocimiento de la situación con la acción adecuada, demuestra capacidad analítica elevada e intuitiva ante situaciones nuevas. ${ }^{17}$

Respecto a la verificación de la adecuada función primaria del corazón, la actividad eléctrica cardiaca se recolecta a través de una serie de cables conectados a la superficie corporal del individuo y esta señal eléctrica se envía a un amplificador que aumentará la pequeña diferencia de potencial que se ha producido en el músculo cardiaco; por ello, la lectura correcta y completa de un trazo electrocardiográfico es la mejor garantía para hacer un diagnóstico correcto. ${ }^{18} \mathrm{El}$ electrocardiograma es una representación de un conjunto de 12 derivaciones, que nos dan una información global y espacial de la actividad eléctrica del corazón. ${ }^{19}$

El objetivo general de este trabajo fue correlacionar el nivel de conocimiento sobre técnica del electrocardiograma con su interpretación básica por estudiantes de Enfermería. 


\section{Material y métodos}

Estudio cuantitativo correlacional, no experimental, prolectivo transversal y prospectivo. Llevado a cabo con un universo de trabajo de 96 estudiantes de la licenciatura en Enfermería de séptimo y octavo semestre de la Universidad Autónoma de Aguascalientes durante el semestre enero-junio 2019, ya que, con base en el programa educativo, se encuentran más avanzados en cuestión de conocimientos en comparación a otros semestres anteriores, se excluyeron a los estudiantes irregulares, a los que no desearon participar o los que no se encontraron al momento de la aplicación del estudio, eliminando así a los estudiantes que abandonaron el cuestionario al momento de la aplicación, que no firmaron el consentimiento informado o que hayan proporcionado instrumentos incompletos.

Las variables utilizadas en ambos instrumentos son de tipo categórica ordinal. En el estudio que determinó el nivel de conocimiento sobre técnica de electrocardiograma, se utilizó la siguiente clasificación: excelente 9-10, bueno 7-8.9, suficiente 6-6.9 e insuficiente con una calificación de 5.9 o menor a ésta.

La investigación fue basada en la teoría de Patricia Benner. El instrumento para identificar el grado de interpretación en la que se encuentran los estudiantes de enfermería de séptimo y octavo semestre de la Universidad Autónoma de Aguascalientes se clasificó de la siguiente manera: principiante $(0-5.9)$, principiante-avanzada (6 7.4), competente (7.5 - 8.4), eficiente (8.5 -9.4) y experta $(9.5-10)$.

Se realizó un proceso de validación de ambos instrumentos, mediante una prueba piloto con 20 estudiantes de otra institución educativa del estado, ahí se explicó la forma de participar contestando el instrumento y aclararon dudas al respecto, posterior a ello se firmó el consentimiento informado. Una vez que se contestaron todos los instrumentos y se recogieron los mismos, se les agregó un folio y se creó la base de datos utilizando un paquete estadístico en la cual se vaciaron los datos, posteriormente se realizó un análisis detallado de cada una de las preguntas y se realizó la obtención de la confiabilidad de cada una de las preguntas y del instrumento en general, obteniendo como confiabilidad un .876 de alfa de Cronbach.

Posteriormente se procedió a aplicar los instrumentos de medición mediante cuestionarios por medio de un censo a 96 estudiantes, con la previa consideración de los criterios de estudio para recabar la información necesaria, y finalmente tras analizar los datos utilizando un paquete estadístico se obtuvieron los resultados.

Para la medición del nivel de conocimiento sobre técnica del electrocardiograma se realizó un instrumento exprofeso titulado "INCOTEECG" 20 por los autores; Hurtado, Moreno, Padilla, Reyes y Rojas dicho instrumento consta de 3 apartados: I. Anatomía y Fisiología del Sistema Cardiovascular, II. Electrocardiograma y III. Técnica del electrocardiograma, conteniendo 19 ítems; validado previamente por enfermera intensivista y la asesora metodológica y disciplinar de la presente investigación, al someter a una prueba piloto con participantes similares a la población de estudio, se encontró un nivel de fiabilidad de 0.75 de alfa de Cronbach.

En el instrumento sobre la interpretación básica del electrocardiograma también se realizó el instrumento de Martínez, Hurtado y Rodríguez (2015) ${ }^{14}$ con un nivel de confiabilidad de 0.86. Consta sólo del apartado de interpretación, el cual contiene 25 ítems, obteniendo un nivel de fiabilidad de 0.876 de alfa de Cronbach en la prueba piloto. Llevando el mismo proceso de validez que el instrumento anterior.

Previo al análisis inferencial se realizó la prueba de Kolmogórov-Smirnov para determinar la distribución de los datos, los cuales presentaron que no existe una distribución normal, por lo que se decidió realizar estadístico de prueba no paramétrica Rho de Spearman.

Se tomaron en cuenta para la presen- 
te investigación las consideraciones éticolegales de la "Declaración de Ginebra de la Asociación Médica Mundial", la Ley General de Salud con el artículo 13, artículo 16, artículo, artículo 23, el Código de Ética para enfermeras haciendo pleno ejercicio del capítulo $v$ de los deberes de las enfermeras para con su profesión; artículo vigésimo cuarto y los principios éticos como lo son el principio de autonomía, no maleficencia, beneficencia y justicia.

\section{| | | | | | | | | | | | | | | | | | | | | | | | | | | | | | | | | | | | | | | | | | | | | | | | | | | | | | | | | | | | | | | | | | | | | | | | | | | | | | | | | | | | | | | | | | | | | | | | | | | | | | | | | | | | | | | | | |}

\section{Resultados}

Quedaron incluidos 96 estudiantes de la carrera de enfermería inscritos en el semestre enero-junio 2019 en la Universidad Autónoma de Aguascalientes. Predominó el sexo femenino en el $77 \%$, con un rango de edad entre los 19 y 28 años, la mayor parte de los estudiantes estuvieron en el rango de 19 a 21 años (47\%); así mismo, más de la mitad de los estudiantes (55\%) estuvieron inscritos en el octavo semestre de la carrera.

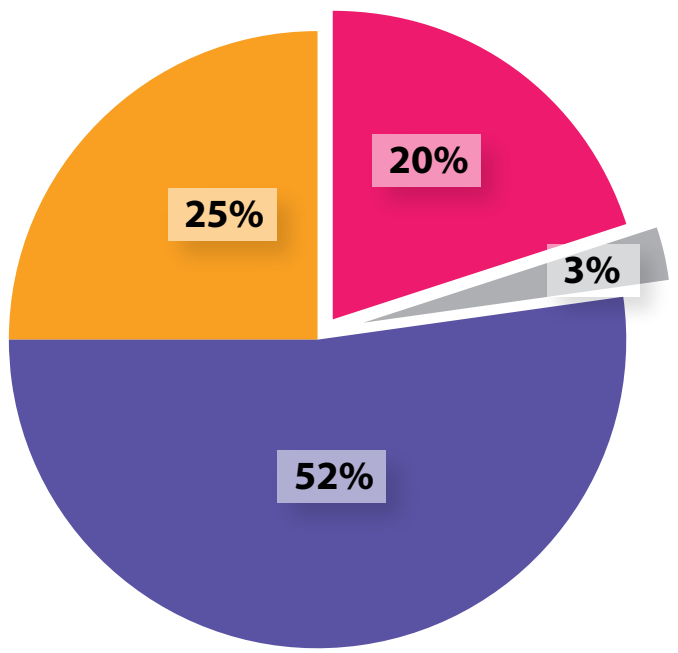

En su mayoría, los estudiantes de la licenciatura en Enfermería obtuvieron un grado de interpretación principiante-avanzado de acuerdo con la clasificación de Patricia Benner. Le siguieron en orden descendente el principiante, el competente,
La mayoría de los estudiantes que participaron en el estudio, obtuvieron un nivel "bueno" en relación con sus conocimientos sobre la técnica del electrocardiograma $(52 \%)$, seguido de suficiente, insuficiente y excelente en el $25 \%, 20 \%$ y $3 \%$, respectivamente. Es de llamar la atención que uno de cada cinco estudiantes evaluados tiene un nivel insuficiente y sólo un 3\% fue excelente (figura 1).
Figura 1. Nivel de conocimientos sobre técnica de electrocardiograma por estudiantes de la licenciatura en Enfermería $(n=96)$ ambos con el $20 \%$ y el eficiente con sólo el $7 \%$ (figura 2).

No encontramos significancia estadística entre el nivel de conocimientos sobre técnica del electrocardiograma con la interpretación básica por estudiantes de Enfer- 


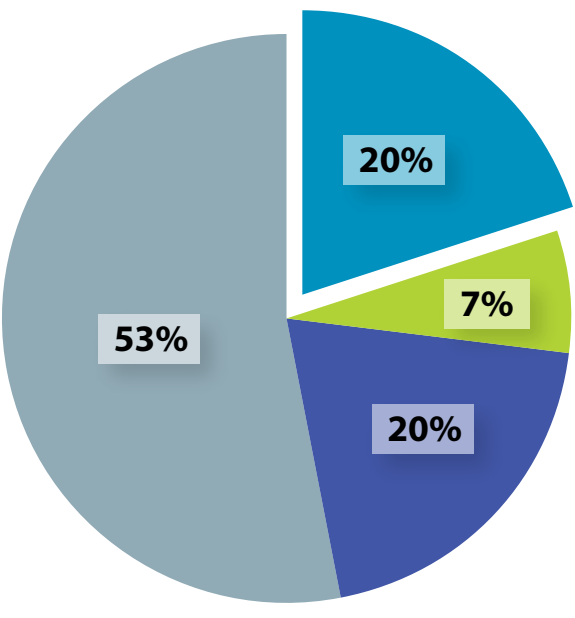

mería (tabla 1). Por lo tanto, un estudiante de la licenciatura en Enfermería puede tener un conocimiento excelente en la técni- ca del electrocardiograma y tener un grado de principiante en interpretación básica de un trazo electrocardiográfico o viceversa.

\section{Tabla I}

Correlación entre nivel de conocimiento e interpretación básica del electrocardiograma y significancia estadística

\begin{tabular}{|l|c|c|c|}
\hline & & & Grado interpretación \\
\hline Rho Spearman & Nivel conocimiento & Coeficiente de correlación & .183 \\
\hline & & Sig. (bilateral) & .074 \\
\hline
\end{tabular}

Al desglosar los resultados de acuerdo con el semestre al que pertenecen los estudiantes, observamos que no existe diferen- cias significativas entre el nivel de conocimiento y la técnica de electrocardiograma (tabla 2).

\section{Tabla 2}

Distribución del nivel de conocimiento de los estudiantes evaluados, de acuerdo con el semestre en que están inscritos

\begin{tabular}{|c|c|c|c|c|c|c|}
\hline \multicolumn{7}{|c|}{ Nivel de conocimiento sobre el ECG $(n=96)$} \\
\hline & & \multicolumn{4}{|c|}{ Nivel conocimiento } & \multirow[t]{2}{*}{ Tota } \\
\hline & & Excelente & Bueno & Suficiente & Insuficiente & \\
\hline \multirow[t]{2}{*}{ Semestre } & Séptimo & 2 & 24 & 6 & 11 & 43 \\
\hline & Octavo & 1 & 26 & 18 & 8 & 53 \\
\hline Total & & 3 & 50 & 24 & 19 & 96 \\
\hline
\end{tabular}


En cuanto a los resultados sobre la interpretación del trazo del ECG, los estudiantes del séptimo obtuvieron mayor número de estudiantes eficientes y competentes comparados con los estudiantes del octavo semestre (tabla 3).

\section{Tabla 3}

\section{Distribución de la interpretación del ECG, en los estudiantes evaluados, de acuerdo con el se- mestre en el que están inscritos.}

\begin{tabular}{|l|l|c|c|c|}
\hline \multicolumn{7}{|c|}{ Nivel de interpretación sobre el ECG $(\mathbf{n = 9 6 )}$} \\
\hline \\
\hline
\end{tabular}

Referente a los resultados arrojados de la teoría básica del corazón, se observó que el $82.8 \%$ de los estudiantes responden correctamente a que se debe revisar el electrocardiograma para corroborar si hace falta repetir el estudio para una adecuada interpretación. Por otro lado, sólo el $1.6 \%$ desconoce las arritmias básicas del corazón que se pueden detectar mediante este estudio no invasivo.

El $85.9 \%$ de los estudiantes conoce cuáles son los elementos principales para que se lleve a cabo la conducción eléctrica cardiaca, por otro lado, sólo el 3.1\% desconoce las partes histológicas básicas del corazón.

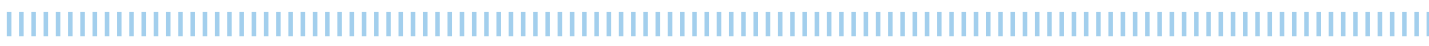

\section{Discusión}

Los resultados obtenidos en este estudio son similares con lo publicado por Martínez, Hurtado y Rodríguez (2015) ${ }^{14}$ que aseguran que el personal de enfermería tiene mayor conocimiento en anatomía del corazón que en la interpretación del electrocardiograma. También se concuerda con Ochoa-Castro ${ }^{11}$ quien encontró una desvinculación entre el conocimiento teórico y la práctica en la interpretación del electrocardiograma. Se coincide con ellos, ya que los estudiantes obtuvieron un nivel de conocimiento bueno en los reactivos que valoraban aspectos de la anatomía y fisiología del corazón, así como en algunas preguntas que se formularon para la técnica en la toma del electrocardiograma, a diferencia del grado de interpretación de la misma técnica.

En lo que respecta a la teoría de Patricia Benner que detalla en De principiante a experta: excelencia y dominio de la práctica de Enfermería clínica, describe las características de la actuación enfermera en las diferentes etapas de adquisición de habilidades y sustenta que la práctica puede ampliar los conocimientos y habilidades del profesional a través del tiempo. 
Se encuentra que un $53 \%$ de los estudiantes de Enfermería que participaron en esta investigación obtuvieron la categoría de principiante-avanzado, es decir, que demuestran experiencia necesaria y una actuación aceptable, sin embargo, aún posee dificultades para dominar determinadas situaciones ante el paciente.
Se observó que los estudiantes de séptimo semestre tienen un grado de interpretación mayor en comparación a los de octavo, por lo que se difiere con Alcántara Guerrero (2016), ${ }^{21}$ el cual menciona que a mayor grado de estudios y mayor la experiencia, existe un aumento en el nivel de conocimientos.

\section{Conclusiones}

La desvinculación entre las variables se debe a un insuficiente abordaje en la técnica e interpretación de un electrocardiograma durante la formación académica. Se concluye que no existe variación significante en el nivel de conocimiento sobre técnica de electrocardiograma según el semestre cursado por lo cual se propone la implementación de este procedimiento diagnóstico no invasivo en los programas educativos de Enfermería desde los primeros semestres y el fortalecimiento en anatomía y fisiología del sistema cardiovascular, fundamentos de Enfermería, urgencias, cuidados intensivos, además de que se incluya como uno de los objetivos principales en la práctica clínica de Enfermería de forma continua, con el fin de permitir que los estudiantes adquieran la experiencia y aprendizaje necesarios para poder brindar cuidados integrales de enfermería a pacientes que requieran de este procedimiento diagnóstico, así como, saber interpretar de manera básica un electrocardiograma. Además, se propone realizar investigaciones futuras referentes al electrocardiograma en la disciplina de Enfermería para detectar los puntos que se han fortalecido a partir de esta investigación en los estudiantes de esta institución y que otros puntos son necesarios reforzar con mas hincapié y que con esto se refuerce la calidad de atención por parte de los estudiantes de la UAA que en un futuro serán los que brindarán atención especializada a la población.

\section{Bibliografía}

1.- OMS, "Enfermedades Cardiovasculares", enero 2015. consultado el 2 de febrero del 2019, sitio web: http:// www.who.int/mediacentre/factsheets/fs317/es/.

2.- OMS, "Prevención de las enfermedades crónicas: una inversión vital" 2009. Ginebra, $\mathrm{CH}$ :. consultado el 2 de febrero 2019, sitio web: http://www.ebrary.com

3.- American Heart Association. "Resumen de Estadísticas de 2017 Enfermedad del corazón y ataque cerebral", 25 de enero 2017. Consultado el 2 de febrero del 2019, sitio web: http://www.heart.org/idc/groups/ahamahpublic/@wcm/@sop/@smd/documents/downloadable/ ucm 491392.pdf

4. Laura Crujillo, García Chong. Orantes Ruiz. "Salud- Enfermedad- Atención en Chiapas", 2014. Consultado el 2 de febrero 2019, sitio web: http://www.espacioimasd.unach. $\mathrm{mx} /$ docs/pdf/doc_academico_medicina.pdf
5.- SEGOB, Secretaria de Gobernación. "Norma Oficial Mexicana Nom-030-Ssa2-2009, Para la Prevención, Detección, Diagnóstico, Tratamiento Y Control De La Hipertensión Arterial Sistémica" 2010. Consultado el 2 de febrero 2019, sitio web: http://dof.gob.mx/nota_detalle. php? codigo $=5144642 \&$ fecha $=31 / 05 / 2010$

6.- Lara Prado J. "El electrocardiograma: una oportunidad de aprendizaje". Revista De La Facultad De Medicina De La UNAM. Noviembre 2016; 59(6): 39-42. Available from: Academic Search Complete.

7.- Lazo Ana María, Ormeño Rosario. "Nivel de conocimiento sobre la valoración del electrocardiograma y su registro en las notas de Enfermería de la unidad coronaria y cuidados intensivos del Hospital Nacional Arzobispo Loayza". 2015. Pág. 4, recuperado el 2 de febrero del 2019, sitio web: http://190.116.48.45/bitstream/ handle/upch/490/Nivel\% 20de\% 20conocimiento\% 20 sobre\% 20la\% 20valoraci\% C3\% B3n\% 20del\% 20electro- 
cardiograma $\% 20 y \% 20$ su $\%$ 20registro $\%$ 20en $\%$ 20las $\% 20$ notas $\%$ 20de \% 20enfermer\% C3\% ADa\% 20de \% 20la\% 20 Unidad \% 20Coronaria \% 20y\% 20Cuidados \% 20Intensivos $\% 20 \mathrm{del} \% 2$ Hospital\% 20Nacional\% 20Arzobispo $\% 20$ Loayza.pdf? sequence $=1$ \&isAllowed $=y$

8.- Sánchez, Bobadilla, Dimas, Gómez, y cols. Enfermedad cardiovascular: primera causa de morbilidad en un hospital de tercer nivel. Revista mexicana de Cardiología julio-septiembre 2016. Consultado el 2 de febrero del 2019, sitio web: https://www.google.com.mx/url?sa $=$ t\&source $=$ we b\&rct=j\&url=http://www.medigraphic.com/pdfs/cardio/ h2016/hs163a.pdf\&ved=0ahUKEwi1maCQnv VAhWD4 CYKHXh1CeMQFggcMAA\&usg=AFQjCNEAUyz7B0y08 BKn r1350KI99Lh9A

9.- OMS. "México: Perfil de Enfermedades Cardiovasculares". 2014. Consultado el 28 de agosto, sitio web: file:///C:/ Users/Usuario/Downloads/MEXICO-PERFIL-ECV-2014 pdf

10.- Ochoa Castro, Pérez Cortez, et al. "Aptitud clínica en la interpretación de electrocardiograma en una muestra de médicos becarios", Facultad de Medicina Universidad Nacional Autónoma de México, 2014, pág. 10, Consultado el 2 de febrero del 2019. Disponible en: http://www.scielo. org. $\mathrm{mx} /$ scielo.php?pid $=$ S200750572014000100003\&scri $\mathrm{pt}=$ sci_abstract

11.-INEGI, Aguascalientes, "Principales causas de mortalidad por residencia habitual, grupos de edad y sexo del fallecido" 2015, Consultado el 2 de febrero del 2019, disponible en: http//www.inegi.org.mx/est/contenidos/proyectos/ registros/vitales/mortalidad/tabulados/ConsultaMortalidad.asp

12.- Secretaria de Salud ISSEA. "Estadísticas - Principales Causas de Mortalidad General" 2010-2015, Consultado el 2 de febrero del 2019, disponible en: http://www.aguascalientes.gob.mx/isea/mortgral.asp

13.- Portal de Gobierno del Estado de Aguascalientes." Enfermedades Crónico Degenerativas" Consultado el 2 de febrero del 2019, sitio web: http://www.aguascalientes. gob.mx/ISEA/progprio1.aspx

14.- Martínez, Hurtado y Rodríguez "nivel de conocimiento en el monitoreo del electrocardiograma por el personal de Enfermería", Aguascalientes 2015. (Tesis)

15.- Página 24. "El IMSS Aguascalientes Implementará un Nuevo Modelo Preventivo de Enfermedades". Aguascalientes 2019. Consultado el 3 de febrero del 2019, sitio web: http://pagina24.com.mx/2017/06/26/local/el-imssaguascalientes-implementara-un-nuevo-modelo-preventivo-de-enfermedades/

16.- El Heraldo. "Las afecciones cardiacas, la primera causa de muerte" Aguascalientes. 2017 consultado el 30 de febrero del 2019, sitio web: http://www.heraldo.mx/las-afecciones-cardiacas-principal-causa-de-muerte/

17.-Dr. Mena, Bermúdez, Calderón y cols. Practica enfermera según la teoría de Patricia Benner: de principiante a experta. 04 agosto 2016. Consultado el septiembre 17. Sitio web: https://www.revista-portalesmedicos.com/revistamedica/teoria-patricia-bennerprincipiante-experta/

18. Ramírez-Ramírez, Jaffet. "Fisiología cardiaca". Revista Médica MD. 2009. Consultado: septiembre del 2017;1(3):4 Disponible en:http://www.medigraphic.com/pdfs/revmed/md-2009/md093d.pdf

19- Lara Prado J. "El electrocardiograma: una oportunidad de aprendizaje". Revista de la Facultad de Medicina de la UNAM. (2016, Nov), Consultado septiembre 2017; 59(6): 39-42. Availablefrom: AcademicSearch Complete.

20- INCOTEECG (enero 2019) y Nivel de conocimientos en el monitoreo del electrocardiograma por el personal de Enfermería (2015). Instrumento exprofeso.

21- Alcántara Guerrero." Conocimientos sobre electrocardiografía en estudiantes de especialidad Enfermería Intensivista". UNMSM 2016. Universidad Nacional mayor de San Marcos. Consultado julio del 2019. Disponible en: http://cybertesis.unmsm.edu.pe/bitstream/handle/cybertesis/7224/Alcantara_gl.pdf? sequence $=1$ \&isAllowed $=y$ 